\title{
Simultaneous Detection of Dual Nucleic Acids Using a SERS-based Lateral Flow Biosensor
}

Article in Analytical Chemistry · November 2016

DOI: 10.1021/acs.analchem.6b03536

CITATION

1

6 authors, including:

\section{Xiaokun Wang}

Hanyang University

7 PUBLICATIONS 114 CITATIONS

SEE PROFILE

\section{Lingxin Chen}

Chinese Academy of Sciences

271 PUBLICATIONS

7,542 CITATIONS

SEE PROFILE
Ziyi Cheng

Hanyang University

8 PUBLICATIONS 53 CITATIONS

SEE PROFILE

Jaebum Choo

Hanyang University

239 PUBLICATIONS 6,175 CITATIONS

SEE PROFILE

Some of the authors of this publication are also working on these related projects:

The Natural Science Research Foundation of China, Grant No. 21575159, "The analysis method for the intracellular active species interactive changes under typical heavy metal ions stress by biological optical imaging technology". View project

The Natural Science Research Foundation of China, Grant No. 21275158, "Study on the construction of functionalized nano optical probes for probing forms of trace heavy metal pollutants in water". View project 


\title{
Simultaneous Detection of Dual Nucleic Acids Using a SERS-Based Lateral Flow Assay Biosensor
}

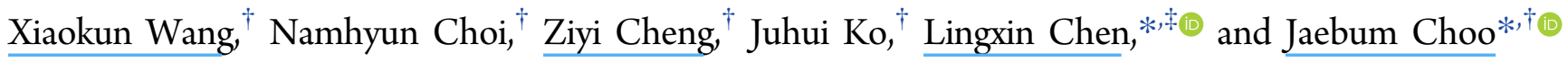 \\ ${ }^{\dagger}$ Department of Bionano Engineering, Hanyang University, Ansan 426-791, South Korea \\ ${ }^{\ddagger}$ Key Laboratory of Coastal Environmental Processes and Ecological Remediation, Yantai Institute of Coastal Zone Research, Chinese \\ Academy of Sciences, Yantai 264003, China
}

Supporting Information

ABSTRACT: A new class of surface-enhanced Raman scattering (SERS)-based lateral flow assay (LFA) biosensor has been developed for the simultaneous detection of dual DNA markers. The LFA strip in this sensor was composed of two test lines and one control line. SERS nano tags labeled with detection DNA probes were used for quantitative evaluation of dual DNA markers with high sensitivity. Target DNA, associated with Kaposi's sarcoma-associated herpesvirus (KSHV) and bacillary angiomatosis (BA), were tested to validate the detection capability of this SERS-based LFA strip. Characteristic peak intensities of SERS nano tags on two test lines were used for quantitative evaluations of KSHV and BA. The limits of detection for KSHV and BA, determined from our SERS-based LFA sensing platform, were estimated to be 0.043 and $0.074 \mathrm{pM}$, respectively. These values indicate approximately 10000 times higher sensitivity than previously reported values using the aggregation-based colorimetric method. We believe that this is the first report of simultaneous detection of two different DNA mixtures using a SERS-based

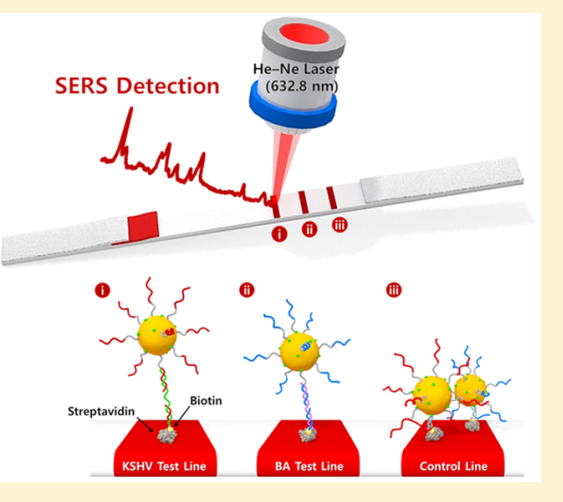
LFA platform. This novel detection technique is also a promising multiplex DNA sensing platform for early disease diagnosis.

$\mathrm{L}$ ateral flow assay (LFA) strips have been considered to be attractive biosensing tools due to their low cost and userfriendly format. ${ }^{1-5}$ These strips have been extensively used in numerous diagnostic applications but they have major limitations in terms of detection sensitivity and limited quantification capability. ${ }^{6-9}$ To resolve these problems, we recently developed a conceptually new surface-enhanced Raman scattering (SERS)-based LFA sensor for highly sensitive and rapid detection of target biomarkers. ${ }^{10,11}$ The detection principle, involving lateral flow chromatographic diffusion and colorimetric detection of gold nanoparticles, is identical to that of conventional naked eye detection-based LFA sensors. The only difference in the SERS-based LFA sensor is the employment of Raman reporter-labeled gold nanoparticles as detection probes. Use of a Raman spectroscopic reader combined with SERS LFA strips enables quantitative evaluation of a specific biomarker with high sensitivity. We recently reported the results of an ultrasensitive assay for Staphylococcal enterotoxin $B^{10}$ and human immunodeficiency virus type 1 (HIV-1) DNA ${ }^{11}$ using this SERS-based LFA sensing platform.

Compared with single biomarker detection, a multiplex biomarker assay enables the simultaneous recognition of multiple target biomarkers in a single assay. Simultaneous multiple biomarker assays provide remarkable advantages including low sample consumption, high sample throughput, and reduced cost per assay. ${ }^{12-16}$ The SERS-based assay platform has been considered a good candidate for multiplex assays due to its narrow bandwidth and nonphotobleaching effect compared with a fluorescence-based detection technique. ${ }^{17-20}$ In the present work, we extensively utilized our SERS-based LFA platform for the simultaneous detection of dual DNA markers. In this SERS-based LFA sensor, the presence of two DNA markers can be identified through color changes of two test lines on nitrocellulose (NC) membrane. Additionally, the amounts of both DNA markers can be simultaneously estimated by measuring the corresponding SERS signal intensities.

In the present study, two target DNAs, associated with Kaposi's sarcoma (KS) and bacillary angiomatosis (BA), were used to validate a dual detection capability of our SERS-based LFA strip. The corresponding genetic targets are Kaposi's sarcoma-associated herpesvirus (KSHV) DNA and bacillary angiomatosis (BA) DNA. KS is known as the most prevalent cancer in patients who are infected with human immunodeficiency virus (HIV). ${ }^{21,22}$ It is particularly important to develop a direct and facile diagnostic method because $\mathrm{KS}$ is one of the leading cancers in the under-developed countries of Africa. ${ }^{23,24}$ Accordingly, a suitable diagnostic implementation for lowresource settings is critically needed. However, BA has very similar clinical presentation and histopathological features to $\mathrm{KS}$, and it is often difficult to distinguish $\mathrm{KS}$ from $\mathrm{BA} .^{25} \mathrm{BA}$ is caused by a rickettsial organism and can be fatal if not treated

Received: September 8, 2016

Accepted: November 22, 2016

Published: November 22, 2016 

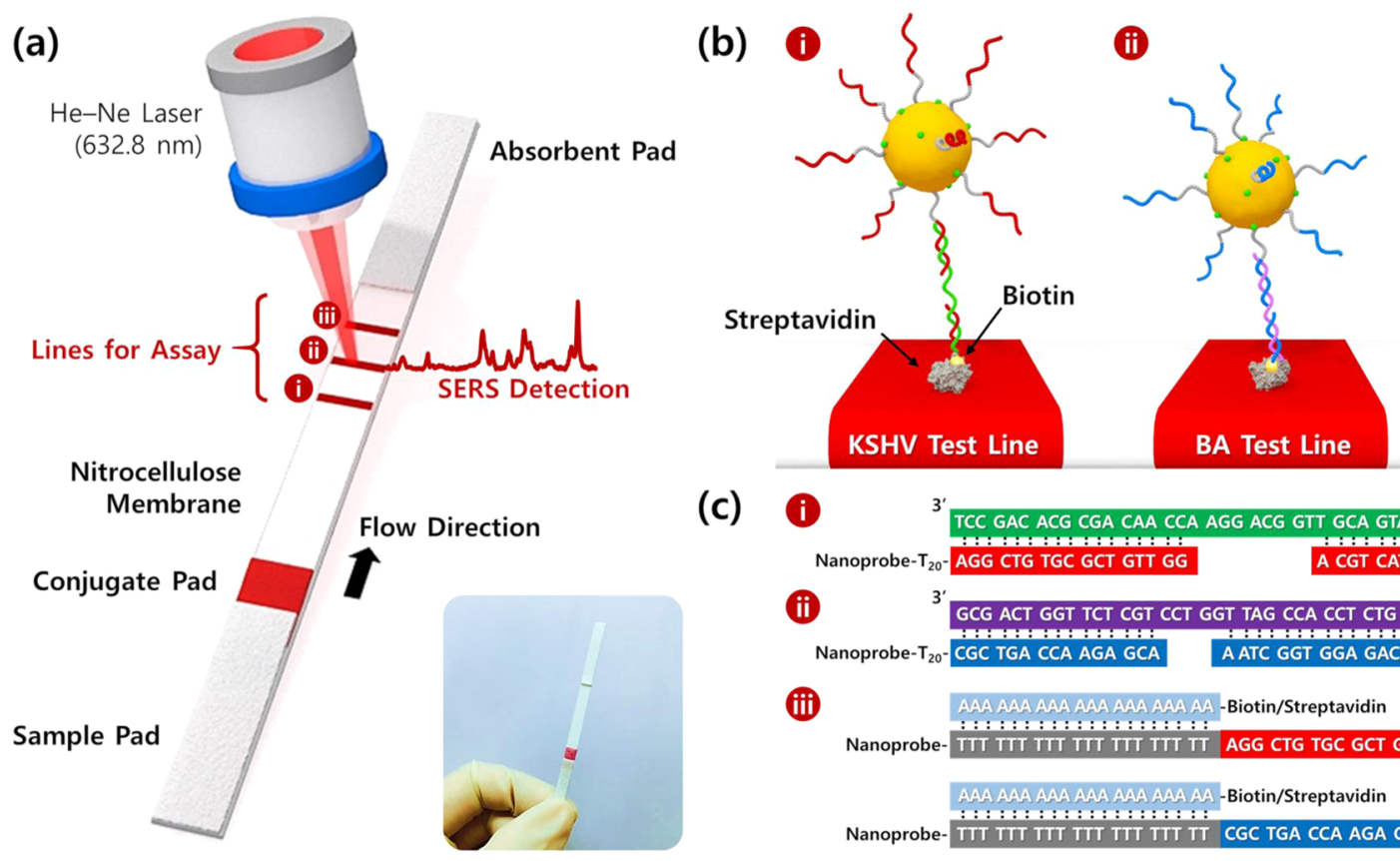

(ii)

(c)

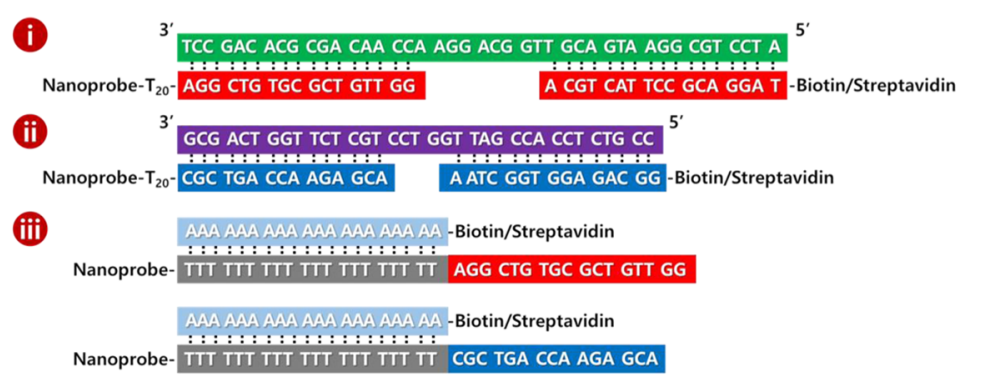

Figure 1. (a) Schematic illustration of the LFA biosensor for the simultaneous detection of two nucleic acids. The strip is composed of two test lines and one control line. (b) (i) KSHV DNA-Au NPs complexes were captured by the probe KSHV DNAs on the first test line; (ii) BA DNA-Au NPs complexes were captured by the probe BA DNAs on the second test line, and (iii) excess KSHV and BA detection DNAs attached to Au NPs were captured by control DNAs through $\mathrm{T}_{20}-\mathrm{A}_{20}$ hybridization on the third control line. (c) Corresponding DNA hybridizations for two test lines (i and ii) and one control line (iii).

with appropriate antibiotic therapy. ${ }^{26,27}$ Therefore, the development of a proper diagnostic technique to differentiate KS from BA is essential for the clinical diagnosis of these two diseases.

Recently, Mancuso et al. reported a colorimetric method for the differential diagnosis of KS and BA. ${ }^{28}$ In this approach, gold and silver nanoparticles aggregations were tuned for each target DNA and a multicolor changing system was developed for the detection of both targets with sensitivity of $1 \mathrm{nM}$. This aggregation-based colorimetric detection has some merits including its speed and simplicity but further improvements in sensitivity and the capability for quantitative analysis are still needed for the early diagnosis of KS and BA. To resolve these problems, we utilized a SERS-based LFA platform for highly accurate and reproducible analysis of these two different types of DNA. To the best of our knowledge, this is the first report of simultaneous detection of two different target DNAs using a SERS-based LFA strip platform.

\section{EXPERIMENTAL SECTION}

Materials and Chemicals. Gold(III) chloride trihydrate $\left(\mathrm{HAuCl}_{4}\right)$, trisodium citrate $\left(\mathrm{Na}_{3}\right.$-citrate), tris (2-carboxyethyl) phosphine hydrochloride (TCEP), sodium dodecyl sulfate (SDS), $\mathrm{Na}_{3} \mathrm{PO}_{4} \cdot 12 \mathrm{H}_{2} \mathrm{O}$, sucrose, Tris- $\mathrm{HCl}$ buffer ( $\mathrm{pH} 7.0$ ), saline-sodium citrate (SSC) buffer ( $\mathrm{pH} 7.0$ ), hydroxyethyl piperazineethanesulfonic acid (HEPES) buffer (1.0 M), 4morpholineethanesulfonic acid (MES) buffer, streptavidin and bovine serum albumin (BSA) were purchased from SigmaAldrich (St. Louis, MO, USA). Phosphate-buffered saline (PBS, $\mathrm{pH} 7.4,0.01 \mathrm{M}$ ), malachite green isothiocyanate (MGITC) and Quant-iT PicoGreen dsDNA Assay Kit were purchased from Life Technologies (Eugene, OR, USA). TRITON X-100 was purchased from Amresco (Cleveland, OH, USA). The nitrocellulose (NC) membrane attached to a backing card (Hi-flow plus HF90, HF120, HF180) was purchased from
Millipore Corporation (Billerica. MA, USA). Absorbent pads (CF4) were purchased from Whatman-GE Healthcare (Pittsburgh, PA, USA). The probe and target DNA oligonucleotide probes used in this study were purchased from Integrated DNA technologies, Inc. (Coralville, IA, USA) and had the following sequences: ${ }^{29}$ target DNA (KHSV), 5'ATCCTGCGGAATGACGTTGGCAGGAACCAACAGCGCACAGCCT-3'; detection DNA (KHSV), 5'-SH- $\left(\mathrm{CH}_{2}\right)_{6}$-TTTTTTтTTTTTTTTTTTTTAGGCTGTGCGCTGTTGG-3'; capture DNA (KHSV), 5'-ACGTCATTCCGCAGGAT-biotin3'; target DNA (BA), 5'-CCGTCTCCACCGATTGGTCCTGCTCTTGGTCAGCG-3'; detection DNA (BA), 5'-SH$\left(\mathrm{CH}_{2}\right)_{6}$-TTTTTTTTTTTTTTTTTTTTCGCTGACCAAGAGCA-3'; capture DNA (BA), 5'-AATCGGTGGAGACGGbiotin- $3^{\prime}$; and control DNA, 5'-biotin-AAAAAAAAAAAAAAAAAAAA-3'.

The ultrapure water $\left(18 \mathrm{M} \Omega \cdot \mathrm{cm}^{-1}\right)$ used in this work was obtained from a Milli- $Q$ water purification system (Millipore Corporation, MA, USA). All chemicals used in this study were analytical reagent grade.

Instrumentation. UV/visible absorption spectra were obtained with Cary 100 spectrophotometer (Varian, Salt Lake City, UT, USA). High-magnification transmission electron microscopy (TEM) images were acquired using a JEOL JEM $2100 \mathrm{~F}$ instrument (JEOL, Tokyo, Japan) at an accelerating voltage of $200 \mathrm{kV}$. Scanning electron microscopy (SEM) images were obtained with a MIRA3 instrument (TESCAN, Brno, Czech Republic) at an accelerating voltage of $15.0 \mathrm{kV}$. Dynamic light scattering (DLS) data were obtained with a Nano-ZS90 apparatus (Malvern Instruments, Malvern, UK). Raman spectra for the test and control lines of the SERS-based LFNAB strip were acquired using an inVia Renishaw Raman microscope system (Renishaw, New Mills, UK). Fluorescence assay was performed using a microplate reader (Power Wave 
(a)

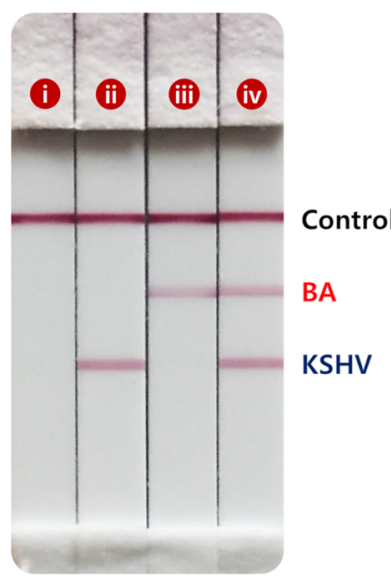

(b)
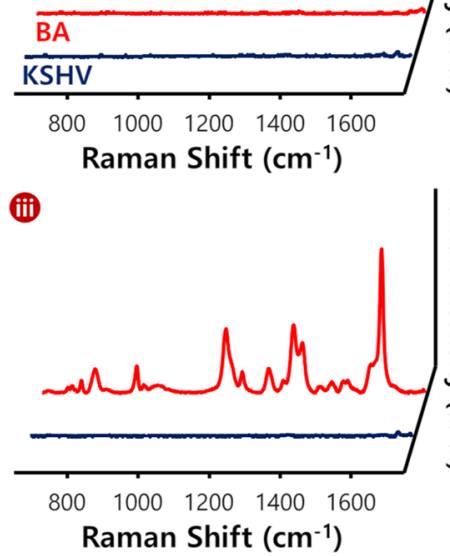

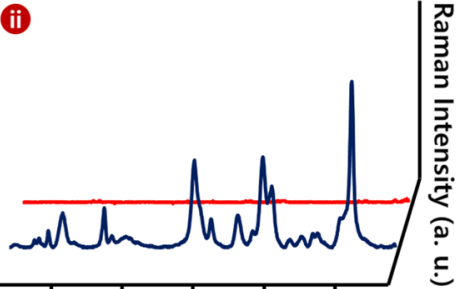

8001000120014001600

Raman Shift $\left(\mathrm{cm}^{-1}\right)$

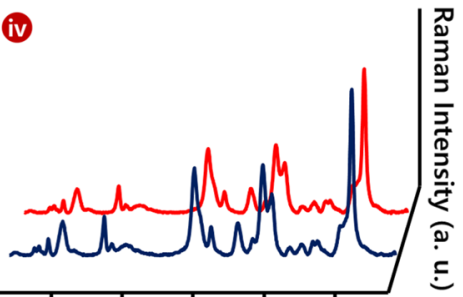

$80010001200 \quad 14001600$

Raman Shift $\left(\mathrm{cm}^{-1}\right)$

Figure 2. (a) Digital photographic images and (b) corresponding SERS spectra of the SERS-based LFA biosensor in the presence of (i) KSHV, 0 pM; BA, 0 pM; (ii) KSHV, 100 pM; BA, 0 pM; (iii) KSHV, 0 pM; BA, 100 pM; (iv) KSHV, 100 pM; BA, 100 pM. Assay time: 20 min.

X340, Bio-Tek, Winooski, VT, USA) equipped with a 96-well plate. Capture and control DNA was immobilized using the DCI 302 dispenser (ZETA Corporation, Seoul, South Korea). All strips were prepared using a programmable cutter (GCI800, ZETA Corporation, Seoul, South Korea).

Preparation of Gold Nanoparticles (AuNPs). Gold NPs were prepared according to the kinetically controlled seed growth method with slight modifications. ${ }^{30}$ Briefly, for the synthesis of Au seeds, a solution of $2.2 \mathrm{mM}$ sodium citrate in a three-necked round-bottomed flask was heated under vigorous stirring. A condenser and thermometer were used to prevent evaporation of the solvent and to control the temperature, respectively. After boiling had commenced, $0.5 \mathrm{~mL} \mathrm{HAuCl}_{4}(25$ $\mathrm{mM}$ ) was added. The color of the solution changed from yellow to bluish gray and then to soft pink over $10 \mathrm{~min}$. The reaction was cooled in the same vessel until the temperature of the solution reached $90^{\circ} \mathrm{C}$, and then $0.5 \mathrm{~mL}$ sodium citrate $(60$ $\mathrm{mM})$ and $0.5 \mathrm{~mL} \mathrm{HAuCl}(25 \mathrm{mM})$ were sequentially added (time delay $\sim 2 \mathrm{~min}$ ). This process was repeated 12 times. The resultant solution was stirred at $90{ }^{\circ} \mathrm{C}$ for $30 \mathrm{~min}$. The final concentration of $\mathrm{Au}$ NPs was estimated to be $\sim 5 \mathrm{nM}$. TEM, UV-vis spectrophotometry, and DLS were used to characterize the morphology and size distribution of the produced Au NPs (Figures S1a-c). A sequential procedure for the immobilization of Raman reporter molecules on the surface of Au NPs was reported elsewhere. ${ }^{19}$ Briefly, $0.6 \mu \mathrm{L}$ of $10^{-4} \mathrm{M}$ MGITC was added to $1 \mathrm{~mL}$ of $0.1 \mathrm{nM}$ Au NPs. The MGITC-Au NPs mixture was reacted for $30 \mathrm{~min}$ under gently stirring. Immobilization of MGITC on the surface of Au NPs was identified using the SERS measurements (Figure S1d).

Preparation and Characterization of DNA-conjugated SERS Nano Tags. Immobilization of thiol-modified DNA onto $\mathrm{Au}$ NPs occurs through covalent bonding between $\mathrm{Au}$ and the terminal thiol group. ${ }^{31}$ Prior to use, the disulfide groups on the DNA were cleaved by the addition of TCEP and incubation at room temperature for $1 \mathrm{~h}$. The solution of DNA with free thiol groups was added to $1 \mathrm{~mL}$ of $0.1 \mathrm{nM}$ MGITC-Au NPs solution, and the concentrations of Tris- $\mathrm{HCl}$ and SDS were adjusted to $0.01 \mathrm{M}$ and $0.01 \%$, respectively. The mixture was maintained at room temperature for $30 \mathrm{~min}$. Subsequently, the concentration of $\mathrm{NaCl}$ was increased to $0.2 \mathrm{M}$ using $0.01 \mathrm{M}$ Tris- $\mathrm{HCl}$ containing $2 \mathrm{M} \mathrm{NaCl}$ and $0.01 \%$ SDS, and the solution was incubated for $24 \mathrm{~h}$ at room temperature. Unbound DNAs and chemicals were removed by centrifugation at $7000 \mathrm{rpm}$ for 10 min, and the supernatant was discarded. The remaining red pellet was washed three times, and redispersed in $100 \mu \mathrm{L}$ of buffer containing $20 \mathrm{mM} \mathrm{Na}_{3} \mathrm{PO}_{4}, 5 \%$ BSA, $0.25 \%$ Tween 20, and $10 \%$ sucrose.

Preparation of the SERS-Based DNA Probe-Immobilized Lateral Flow Strip. A schematic diagram of the SERSbased LFA sensor prepared in this work is shown in Figure 1a. The SERS-based LFA sensor consisted of four sections: sample pad, conjugate pad, NC membrane, and absorption pad. The sample pad was blocked with $20 \mathrm{mM}$ Tris- $\mathrm{HCl}$ containing $0.25 \%$ TRITON X-100 and $150 \mathrm{mM} \mathrm{NaCl}$, followed by overnight drying at $37^{\circ} \mathrm{C}$ to ensure optimal conditions for the analytes throughout the flux. The conjugated pad was prepared by addition of $6 \mu \mathrm{L}$ of SERS nano tag and dried at room temperature. The NC membrane was used to dispense the streptavidin-biotinylated KSHV capture probe, streptavidinbiotinylated BA capture probe and streptavidin-biotinylated complementary capture probe to form two test lines and one control line, and then cut into $3.8 \mathrm{~mm}$ strips by a programmable cutter. The streptavidin-biotinylated capture probes were prepared by the reaction of $100 \mu \mathrm{L}$ of biotinylated DNA and $100 \mu \mathrm{L}$ of $2 \mathrm{mg} / \mathrm{mL}$ streptavidin at room temperature for $1 \mathrm{~h}$. The resulting conjugates were purified by centrifugation for $30 \mathrm{~min}$ with a centrifugal filter at 6000 rpm and then dissolved in PBS solutions. Finally, all of the components were assembled on a plastic adhesive backing layer. Each part overlapped $2 \mathrm{~mm}$ to ensure solution migration.

\section{RESULTS AND DISCUSSION}

Principle of SERS-Based Lateral Flow Assay for the Simultaneous Detection of Dual DNA Markers. Figure 1 illustrates the operating principle of the LFA biosensor for the simultaneous detection of two nucleic acid biomarkers, KSHV and BA. This strip was composed of two test lines and one control line as shown in Figure 1a. Biotinylated KSHV capture 
(a)

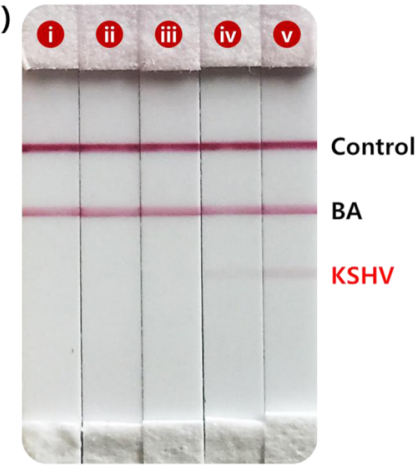

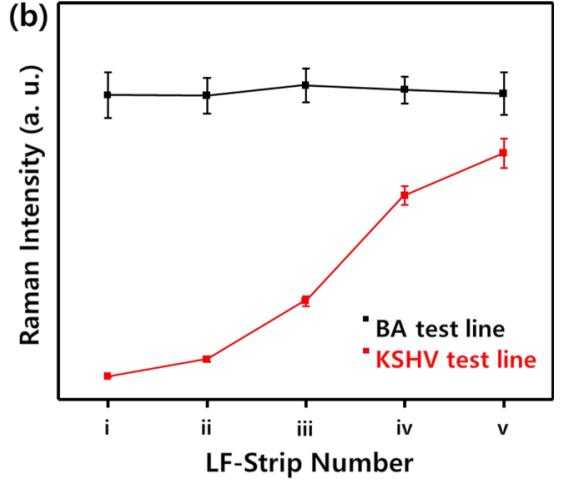

Figure 3. (a) Digital photographic images and (b) corresponding Raman intensity variations at $1617 \mathrm{~cm}^{-1}$ of two test lines (BA and KSHV) in the presence of (i) KSHV, 0 pM; BA, 100 pM; (ii) KSHV, 0.5 pM; BA, 100 pM; (iii) KSHV, 2 pM; BA, 100 pM; (iv) KSHV, 10 pM; BA, 100 pM; (v) KSHV, 20 pM; BA, 100 pM. Assay time: $20 \mathrm{~min}$. The error bars indicate the standard deviations calculated from five measurements.

DNAs were immobilized on the first test line through the streptavidin-biotin reaction. BA capture DNAs were immobilized on the second test line in the same manner. On the control line in the third position, $\mathrm{A}_{20}$-biotinylated capture DNAs were immobilized for direct hybridization with $\mathrm{T}_{20^{-}}$ detection DNAs attached to Au NPs. All capture DNAs and control DNAs were preimmobilized on the NC membrane using the DCI 302 dispenser. At the same time, a 1:1 molar mixture of KSHV and BA detection DNA-immobilized SERS nano tags was stored in the conjugate pad. Detection DNAs were attached on the surface of Au NPs through the covalent bond between thiol and Au. Here, $T_{20}$ DNA spacers were used for both KSHV and BA detection DNAs for simultaneous hybridizations with preimmobilized $\mathrm{A}_{20}$ capture DNAs on the control line. After introduction of the sample solution containing the target DNAs (KSHA and BA) onto the sample pad, the solution migrated toward the absorption pad by capillary action. When the sample solution passed through the conjugation pad, the target DNAs hybridized with corresponding detection DNAs (KSHA and BA) attached to the surface of $\mathrm{Au}$ NPs to form two different types of DNA hybridization complexes, which continued to flow along the strip.

When these complexes reached the first test line, KSHV DNA-Au NPs complexes were captured by the probe KSHV DNAs that were preimmobilized on the test line (Figure $1 \mathrm{~b}(\mathrm{i}$ ) and Figure $1 \mathrm{c}(\mathrm{i}))$. Consequently, sandwich KSHV DNA hybridization complexes were formed on the first test line. The remaining solution continued to migrate, and BA DNA-Au NPs complexes were captured by the probe BA DNAs attached to the second test line (Figure $1 \mathrm{~b}$ (ii) and Figure 1c(ii)). Finally, excess KSHV and BA detection DNAs attached to Au NPs continued to migrate, and then they were captured by the control DNAs through $\mathrm{T}_{20}-\mathrm{A}_{20}$ hybridization on the third control line (Figure $1 \mathrm{~b}$ (iii) and Figure $1 \mathrm{c}(\mathrm{iii})$ ).

"On-Off" Test of KSHA and BA DNA Markers Using SERS-based Lateral Flow Assay. Figure 2a shows digital photographic images of the LFA biosensor for the simultaneous detection of dual DNA markers, and their corresponding color changes for different concentrations of KSHA and BA DNA markers. SERS spectra of two test lines for corresponding four lateral flow strips were displayed in Figure 2b. An "on-off" qualitative test could be performed by observing color changes of two test lines: one for KSHV and the other for BA. It is worth noting that the color of the third control line always changes to red regardless of the concentration of DNA marker, indicating that the control line is working properly by capturing
DNA-conjugated SERS nano tags through $\mathrm{T}_{20}-\mathrm{A}_{20}$ hybridization. If the red band is not observed on the control line, the lateral flow strip is not functioning properly. The colors of the first and second test lines did not change when the sample did not include any DNA markers (Figure $2 \mathrm{a}(\mathrm{i})$ ). Moreover, the color change of the test lines depended on the concentration of each DNA marker. When the sample contained 100 pM KSHA DNA and 0 pM BA DNA, the color of the first test line changed to red (Figure 2a(ii)); when the sample contained 0 pM KSHA and 100 pM BA, the color of the second test line changed to red (Figure 2a(iii)). When the sample included 100 pM KSHA DNA and 100 pM BA DNA, the color of both lines changed to red (Figure 2a(iv)). SERS spectral changes in Figure $2 \mathrm{~b}$ demonstrate the same "on-off" pattern according to the concentration of each DNA. SEM images for the corresponding "on-off' conditions of KSHA and BA are displayed in Figure S2. Therefore, it can be concluded that a qualitative "on-off" identification of two different DNA markers is possible using this SERS-based lateral flow strip.

To evaluate the cross-reactivity between the two DNA markers, $100 \mathrm{pM} \mathrm{BA}$ was mixed with different concentrations of $\mathrm{KSHV}$ in the $0-20 \mathrm{pM}$ range. Photographic images of the detection results are displayed in Figure 3a. As expected, the color of the second test line changed to the same intensity of red color for $100 \mathrm{pM} \mathrm{BA}$ in all samples. The results demonstrate that the intensity variation for $100 \mathrm{pM} B A$ showed consistent values regardless of KSHV concentration. In contrast, the color of the first test line changed to a light brown color for 20 pM KSHV (Figure $3 \mathrm{a}(\mathrm{v})$ ) but no obvious color change was observed when the concentration of KSHV was lower than $20 \mathrm{pM}$. However, the corresponding Raman intensity at $1617 \mathrm{~cm}^{-1}$ exactly correlated with the concentration of KSHV below $20 \mathrm{pM}$, as shown in Figure $3 \mathrm{~b}$. Therefore, quantitative analysis in the lower concentration range of KSHV is possible using this SERS-based assay strip.

Optimization of SERS-Based Lateral Flow DNA Assay Parameters. To achieve optimized conditions for the sandwich-type DNA hybridization reactions and SERS detection of KSHV and BA DNA markers, various experimental parameters, including the NC membrane type, the type and concentration of running buffer and the amount of BSA in the running buffer, were investigated. Optimization was carried out by varying one parameter while keeping the other parameters constant.

Effect of Different Types of Nitrocellulose Membrane. Three different types of NC membranes, HF090MC 100, 
(a)
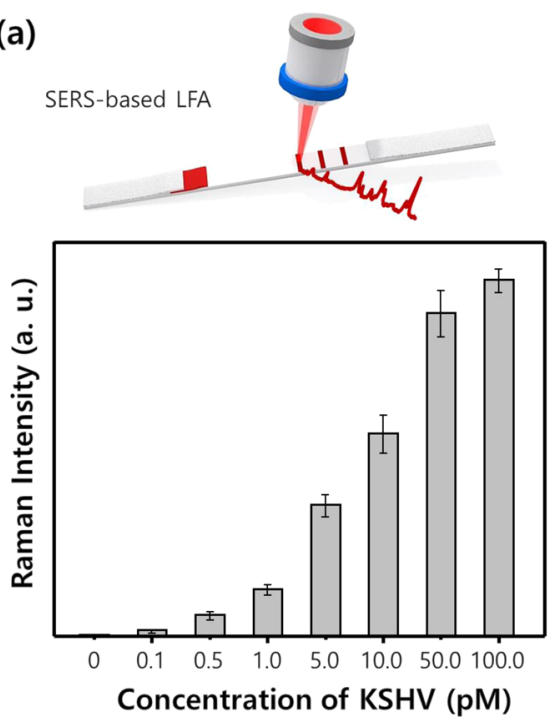

(b)

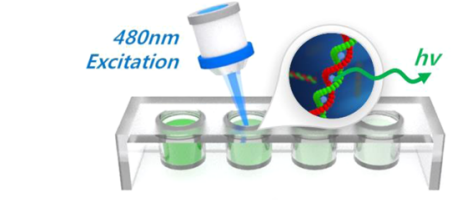

Quant-iT'M PicoGreen ${ }^{\mathbb{R}}$

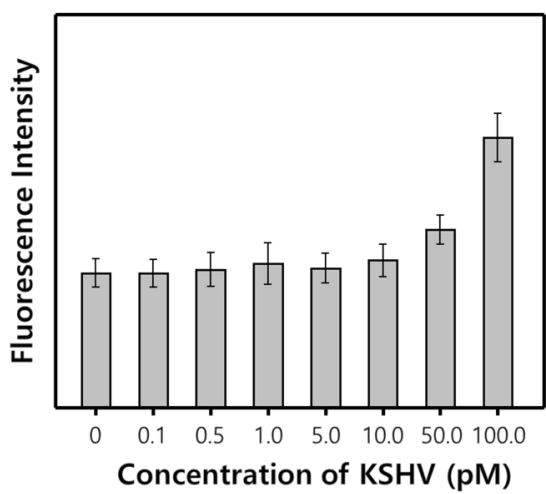

Figure 4. Comparison of the assay results obtained for eight different concentrations of KSHV DNA using (a) the SERS-based lateral flow biosensor and (b) commercially available Quant-iT PicoGreen double strand DNA Assay Kit. The error bars indicate the standard deviations calculated from five measurements.

(a)

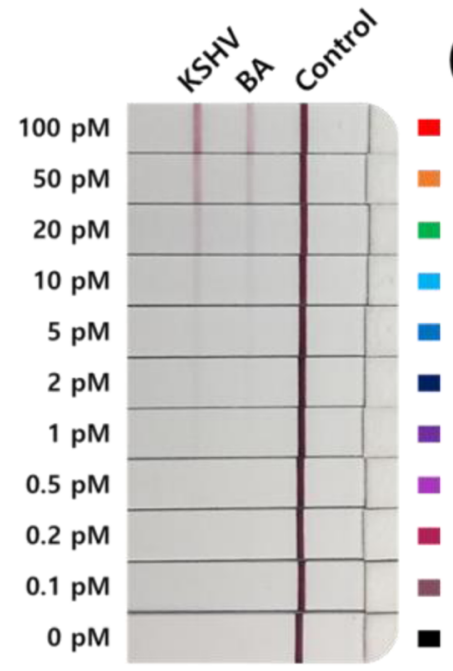

(c)

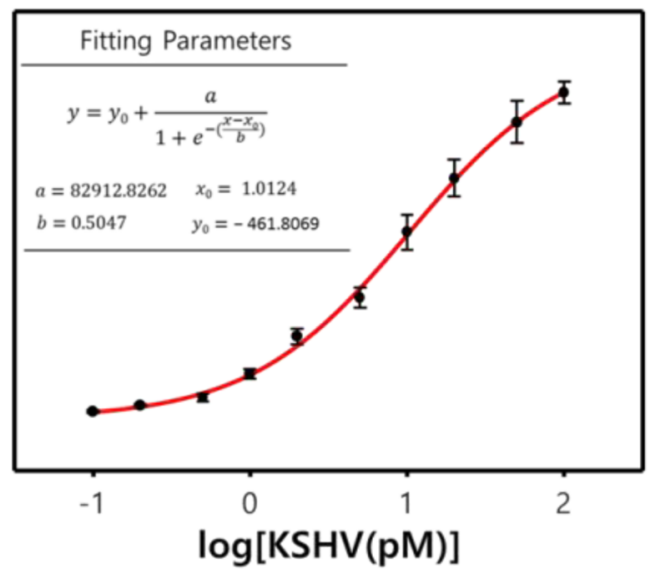

(b)
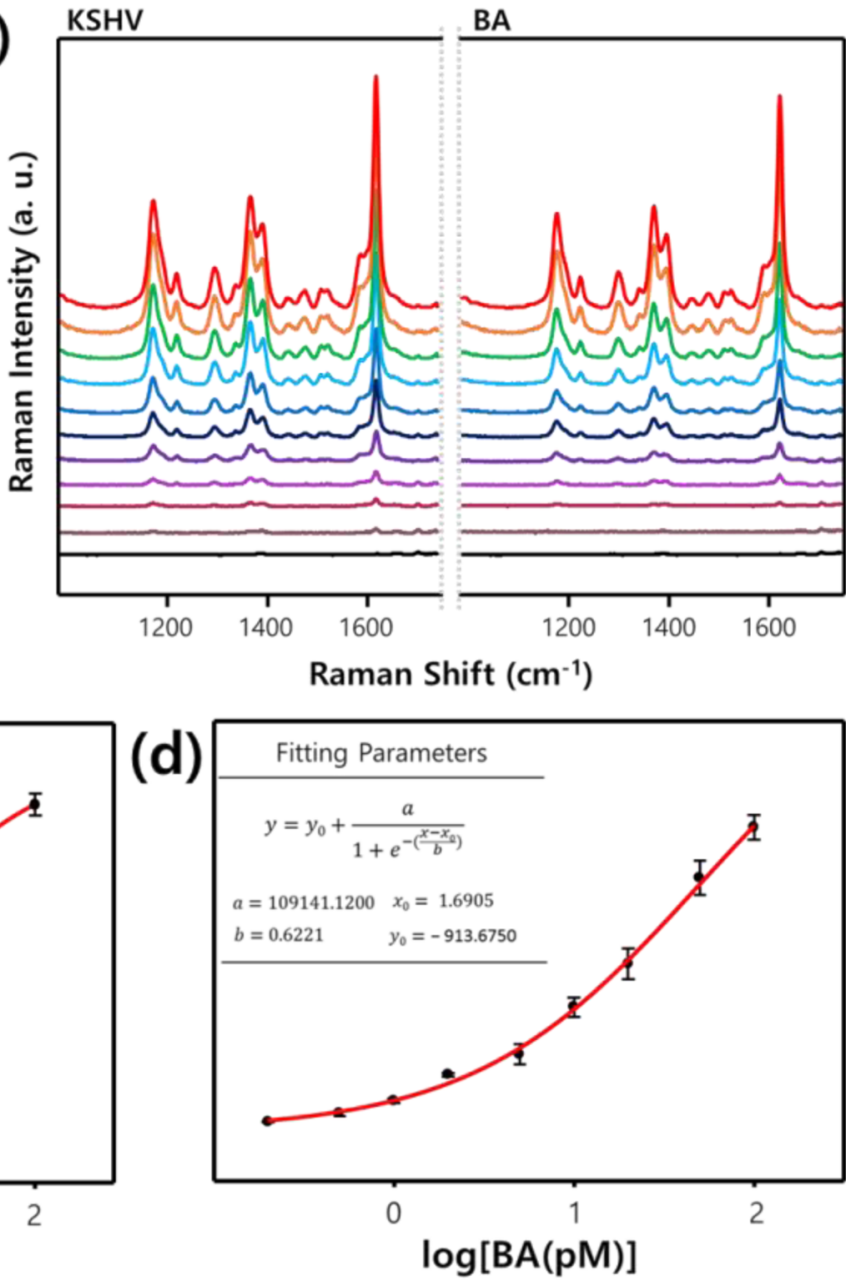

Figure 5. (a) Digital photographic images and (b) corresponding Raman spectra of two test lines for different concentrations of KSHV and BA DNAs (from 0 to $100 \mathrm{pM}$ ). Corresponding calibration lines as a function of the sigmoidal of the concentration of (c) KSHV DNA (from 0.1 to 100 $\mathrm{pM}$ ) and (d) BA DNA (from 0.2 to $100 \mathrm{pM}$ ). Inset denotes the four-parameter sigmoid function for the fitting lines. The error bars indicate the standard deviations calculated from five measurements. 
HF120MC 100, and HF180MC 100, were tested to determine which gave the best SERS response. Figure S3a displays corresponding Raman intensities of KSHV and BA test lines at $1617 \mathrm{~cm}^{-1}$ for three NC membranes. As shown in this figure, the Raman intensity for HF180MC 100 was significantly stronger than those measured for other membranes. According to the manufacturer's instruction, the migration times of the buffer in HF090MC100, HF120MC 100, and HF180MC 100 were estimated to be 10,15 , and $20 \mathrm{~min}$, respectively. Our results indicate that the $\mathrm{NC}$ membrane with a longer migration time is most favorable for DNA assays because the running buffer has a longer duration time on each test line for the formation of sandwich DNA complexes. Therefore, HF180MC 100 was selected for subsequent assay experiments.

Effect of Running Buffer. The running buffer solution strongly affects the migration of SERS nano tags. In addition, the choice of appropriate buffer solution would increase the hybridization efficiency of detection DNA, target DNA and capture DNA. Therefore, we evaluated the performance of the lateral diffusion and SERS detection capability with five different types of running buffer solution: MES, HEPES, Tris$\mathrm{HCl}, \mathrm{PBS}$ and SSC. As shown in Figure S3b, the Raman intensity for SSC buffer was significantly stronger than those for other buffer solutions. On the basis of this result, a SSC buffer solution was used in subsequent assays. As the concentration of SSC buffer solution is another important factor, SERS signal intensities for several different SSC concentrations were tested. As shown in Figure S3c, 4X SSC was estimated to be the optimum concentration for the SERS-based assay. Finally, the amount of BSA in the running buffer also plays an important role in reducing the nonspecific absorption of SERS nano tags on the NC membrane. During the optimization process, the flow velocity decreased with an increase in BSA concentration. It was also found that the Raman intensity decreased with increasing BSA concentration as shown in Figure S3d. We believe that the decrease of Raman intensity is caused by the edge effect, which is caused by preferential evaporation at the edges of the membrane sheet. With the viscosity increase of the buffer solution, more SERS nano tags move to edge areas from interior sections. When the lateral flow strip was completely dried, the edges were noticeably more tinted than the center section. To minimize this edge effect, SSC buffer containing $1 \%$ BSA was determined to be the final buffer solution for subsequent experiments.

Analytical Performance of SERS-Based Lateral Flow Assay Biosensor. To evaluate the quantitative detection capability of our SERS-based LFA strip, its analytical performance for KSHV was compared with that of the commercially available KSHV fluorescence kit (Quant-iT PicoGreen dsDNA Assay Kit) as shown in Figure 4. PicoGreen is a sensitive fluorescence nucleic acid stain for quantitating double-stranded DNA in solution. The DNA samples were excited at $480 \mathrm{~nm}$ and the fluorescence emission intensity was measured at 520 $\mathrm{nm}$ using a fluorescence spectrophotometer. The assay results for KSHV were compared in the $0-100 \mathrm{pM}$ range. The results indicate that the SERS-based assay technique (Figure 4a) provides more sensitive quantification of KSHV than the PicoGreen fluorescence kit (Figure $4 b$ ) especially at concentrations lower than $10 \mathrm{pM}$.

Under optimized detection conditions, quantitative evaluations of KSHV and BA DNA markers in the sample was performed using the SERS-based LFA strip. As shown in the digital photographs in Figure $5 \mathrm{a}$, it is difficult to identify colorimetric changes of test lines for both KSHV and BA at concentrations lower than $10 \mathrm{pM}$. Furthermore, quantitative evaluation of colorimetric changes is impossible. Using the SERS-based LFA strip, it is possible to achieve a quantitative result by monitoring the characteristic Raman signal intensity of SERS nano tags. Quantitative analysis of KSHV and BA DNAs was performed by monitoring the Raman peak intensities of the corresponding test lines. Figure $5 \mathrm{~b}$ illustrates the SERS spectra for different concentrations of target KSHV and BA DNAs. As shown in this figure, overall SERS intensities concomitantly increased with increasing concentrations of KSHV and BA.

The corresponding calibration curves are displayed in Figure $5 \mathrm{c}$ and 5d. The SERS peak intensities centered at $1617 \mathrm{~cm}^{-1}$ were used for quantitative evaluation of KSHV and BA. The inset displays the four-parameter sigmoid function for the fitting line. Error bars indicate the standard deviations of five measurements. The limit of detection (LOD) value of each target was estimated by the IUPAC standard method (LOD = $y_{\text {blank }}+3 \times \mathrm{SD}_{\text {blank }}$, where $y_{\text {blank }}$ is the average signal intensity at zero and $\mathrm{SD}_{\text {blank }}$ is the standard deviation of the blank measurements). ${ }^{32}$ Using this equation, the LODs of KSHV and BA DNAs were estimated to be $0.043 \mathrm{pM}$ and $0.074 \mathrm{pM}$, respectively. These LOD values are at least 10,000 times more sensitive than those obtained from the nanoparticle aggregation-based colorimetric method. ${ }^{28}$ Importantly, our SERS-based LFA strip can be used for simultaneous detection of dual DNA markers, KSHV and BA, with high sensitivity.

\section{CONCLUSION}

In the present study, we developed a novel SERS-based LFA biosensor for the simultaneous detection of two DNA markers, KSHV and BA. This SERS-based LFA strip contained two test lines and one control line, and a 1:1 molar mixture of KSHV and BA detection DNA-immobilized SERS nano tags was stored in the conjugate pad. KSHV and BA detection DNAs attached to Au NPs were captured by corresponding probe DNAs on the first and second test lines, and excess Au NPs were captured by control DNAs on the third control line through $\mathrm{T}_{20}-\mathrm{A}_{20}$ hybridization.

Raman peak intensity centered at $1617 \mathrm{~cm}^{-1}$ was monitored, and its variation was used for the quantitative evaluation of KSHV and BA. The LODs of KSHV and BA determined from our SERS-based LFA sensing platform were estimated to be $0.043 \mathrm{pM}$ and $0.074 \mathrm{pM}$, respectively; these values indicate approximately 10000 times greater sensitivity than the previously reported aggregation-based colorimetric method. We believe that this novel SERS-based LFA biosensor is an excellent diagnostic platform for low-resource settings.

\section{ASSOCIATED CONTENT}

\section{S Supporting Information}

The Supporting Information is available free of charge on the ACS Publications website at DOI: 10.1021/acs.analchem.6b03536.

Characterization of SERS nano tags, the SEM images of the SERS-based LFA biosensor and the optimization of SERS-based lateral flow DNA assay parameters (PDF)

\section{AUTHOR INFORMATION}

\section{Corresponding Authors}

*E-mail: 1xchen@yic.ac.cn. Tel.: +86-535-2109130.

*E-mail: jbchoo@hanyang.ac.kr. Tel.: +82-31-400-5201. 


\section{ORCID}

Lingxin Chen: 0000-0002-3764-3515

Jaebum Choo: 0000-0003-3864-6459

Notes

The authors declare no competing financial interest.

\section{ACKNOWLEDGMENTS}

This work was supported by the Korea Health Industry Development Institute (KHIDI) funded by the Ministry of Health and Welfare, Korea through grant number HI16C2129. This work was partially supported by the National Natural Science Foundation of China (grant numbers 21575159 and 21275158).

\section{REFERENCES}

(1) Bahadir, E. B.; Sezginturk, M. K. TrAC, Trends Anal. Chem. 2016, 82, 286-306.

(2) Taranova, N. A.; Berlina, A. N.; Zherdev, A. V.; Dzantiev, B. B. Biosens. Bioelectron. 2015, 63, 255-261.

(3) Chapman, R.; Lin, Y.; Burnapp, M.; Bentham, A.; Hillier, D.; Zabron, A.; Khan, S.; Tyreman, M.; Stevens, M. M. ACS Nano 2015, 9, $2565-2573$.

(4) Hu, J.; Zhang, Z. L.; Wen, C. Y.; Tang, M.; Wu, L. L.; Liu, C.; Zhu, L.; Pang, D. W. Anal. Chem. 2016, 88, 6577-6584.

(5) Luppa, P. B.; Muller, C.; Schlichtiger, A.; Schlebusch, H. TrAC, Trends Anal. Chem. 2011, 30, 887-898.

(6) Wang, C.; Hou, F.; Ma, Y. Biosens. Bioelectron. 2015, 68, 156162.

(7) Shen, G.; Zhang, S.; Hu, X. Clin. Biochem. 2013, 46, 1734-1738.

(8) Oh, Y. K.; Joung, H.; Han, Y. S.; Suk, H.; Kim, M. Biosens. Bioelectron. 2014, 61, 285-289.

(9) Schmit, V. L.; Martoglio, R.; Carron, T. Anal. Chem. 2012, 84, $4233-4236$.

(10) Hwang, J.; Lee, S.; Choo, J. Nanoscale 2016, 8, 11418-11425.

(11) Fu, X.; Cheng, Z.; Yu, J.; Choo, P.; Chen, L.; Choo, J. Biosens. Bioelectron. 2016, 78, 530-537.

(12) Swanson, C.; D'Andrea, A. Clin. Chem. 2013, 59, 641-648.

(13) Xu, Y.; Liu, Y.; Wu, Y.; Xia, X.; Liao, Y.; Li, Q. Anal. Chem. 2014, 86, 5611-5614.

(14) Lee, J. H.; Seo, H. S.; Kwon, J. H.; Kim, H. T.; Kwon, K. C.; Sim, S. J.; Cha, Y. J.; Lee, J. Biosens. Bioelectron. 2015, 69, 213-225.

(15) Crannell, Z.; Castellanos-Gonzalez, A.; Nair, G.; Mejia, R.; White, A. C.; Richards-Kortum, R. Anal. Chem. 2016, 88, 1610-1616.

(16) Lafleur, L.; Stevens, D.; McKenzie, K.; Ramachandran, S.; Spicar-Mihalic, P.; Singhal, M.; Arjyal, A.; Osborn, J.; Kauffman, P.; Yager, P.; Lutz, B. Lab Chip 2012, 12, 1119-1127.

(17) Chon, H.; Lee, S.; Yoon, S. Y.; Lee, E. K.; Chang, S. I.; Choo, J. Chem. Commun. 2014, 50, 1058-1060.

(18) Lee, S.; Chon, H.; Lee, J.; Ko, J.; Chung, B. H.; Lim, D. W.; Choo, J. Biosens. Bioelectron. 2014, 51, 238-243.

(19) Lee, S.; Chon, H.; Yoon, S. Y.; Lee, E. K.; Chang, S. I.; Lim, D. W.; Choo, J. Nanoscale 2012, 4, 124-129.

(20) Wang, Y. Q.; Yan, B.; Chen, L. Chem. Rev. 2013, 113, 13911428.

(21) Gallo, R. C. Science 1998, 282, 1837-1839.

(22) Chang, Y.; Cesarman, E.; Pessin, M. S.; Lee, F.; Culpepper, J.; Knowles, D. M.; Moore, P. S. Science 1994, 266, 1865-1869.

(23) Eltom, M. A.; Jemal, A.; Mbulaiteye, S. M.; Devesa, S. S.; Biggar,

R. J. J. Natl. Cancer Inst. 2002, 94, 1204-1210.

(24) Jemal, A.; Bray, F.; Center, M. M.; Ferlay, J.; Ward, E.; Forman, D. Ca-Cancer J. Clin. 2011, 61, 69-90.

(25) Rosales, C. M.; McLaughlin, M. D.; Sata, T.; Katano, H.; Veno, P. A.; De Las Casas, L. E.; Miranda, R. N. AIDS Patient Care ST. 2002, $16,573-577$.

(26) Caldwell, B. D.; Kushner, D.; Young, B. J. Am. Podiatr. Med. Assoc. 1996, 86, 260-262.

(27) Tappero, J. W.; Koehler, J. E. N. Engl. J. Med. 1997, 337, 1888.
(28) Mancuso, M.; Jiang, L.; Cesarman, E.; Erickson, D. Nanoscale 2013, 5, 1678-1686.

(29) Altschul, S. F.; Madden, T. L.; Schaffer, A. A.; Zhang, J.; Zhang, Z.; Miller, W.; Lipman, D. J. Nucleic Acids Res. 1997, 25, 3389-3402.

(30) Bastús, N. G.; Comenge, J.; Puntes, V. Langmuir 2011, 27, 11098-11105.

(31) Hurst, S. J.; Lytton-Jean, A. K. R.; Mirkin, C. A. Anal. Chem. 2006, 78, 8313-8318.

(32) Cho, J. H.; Han, S. M.; Paek, E. H.; Cho, I. H.; Paek, S. H. Anal. Chem. 2006, 78, 793-800. 